\title{
Ultrasound-Promoted Synthesis of 3-(Thiophen-2-yl)-4,5-dihydro-1H-pyrazole-1- carboximidamides and Anticancer Activity Evaluation in Leukemia Cell Lines
}

\author{
Eric F. S. dos Santos, ${ }^{a}$ Nathália M. Cury, ${ }^{b, c}$ Tainara A. do Nascimento, ${ }^{a}$ Cristiano \\ Raminelli, ${ }^{d}$ Gleison A. Casagrande, ${ }^{a}$ Claudio M. P. Pereira, ${ }^{e}$ Euclésio Simionatto, ${ }^{f}$ \\ José A. Yunes ${ }^{b, c}$ and Lucas Pizzuti*,a \\ ${ }^{a}$ Grupo de Pesquisa em Síntese e Caracterização Molecular do MS, Universidade Federal da \\ Grande Dourados, Rua João Rosa Góes, 1761, 79825-070 Dourados-MS, Brazil \\ ${ }^{b}$ Departamento de Genética e Biologia Molecular, Instituto de Biologia, Universidade Estadual de \\ Campinas, Rua Monteiro Lobato, 255, 13083-970 Campinas-SP, Brazil \\ ${ }^{c}$ Laboratório de Biologia Molecular, Centro Infantil Boldrini, Rua Dr. Gabriel Porto, 1270, \\ 13083-210 Campinas-SP, Brazil \\ ${ }^{d}$ Departamento de Ciências Exatas e da Terra, Universidade Federal de São Paulo, Rua Prof. Artur \\ Riedel, 275, 09972-270 Diadema-SP, Brazil \\ eLaboratório de Lipidômica e Bio-Orgânica, Centro de Ciências Químicas, Farmacêuticas e dos \\ Alimentos, Universidade Federal de Pelotas, Campus Universitário, 96001-900 Pelotas-RS, Brazil \\ ${ }^{f}$ Universidade Estadual de Mato Grosso do Sul, Rua Emilio Mascolli, 275, 79950-000 Naviraí-MS, Brazil

\begin{abstract}
3-(Thiophen-2-yl)-4,5-dihydro-1H-pyrazole-1-carboximidamides were efficiently prepared through a cyclocondensation of thiophenylchalcones with aminoguanidine hydrochloride under ultrasonic conditions in the presence of $\mathrm{KOH}$ and ethanol as a green solvent in short reaction times (15-35 $\mathrm{min}$ ) and good yields (62-95\%). All compounds produced were evaluated against the human Jurkat and RS4;11 acute lymphoblastic leukemia cell lines of T- and B-cell origin, respectively, and the K562 myelogenous leukemia cell line. Six compounds presented half maximal inhibitory concentration $\left(\mathrm{IC}_{50}\right.$ ) values around $15 \mu \mathrm{mol} \mathrm{L}{ }^{-1}$ and five compounds presented $\mathrm{IC}_{50}$ values around $40 \mu \mathrm{mol} \mathrm{L}{ }^{-1}$ for at least one of the three cell lines analyzed. One compound was not significantly cytotoxic, presenting $\mathrm{IC}_{50}$ value $>100 \mu \mathrm{mol} \mathrm{L}{ }^{-1}$.
\end{abstract}

Keywords: amidinopyrazole, pyrazoline, cytotoxic activity, leukemia, ultrasonic irradiation

\section{Introduction}

Amidine functional group has proven to be an important fragment in compounds with recognized bioactivities. In this sense, several amidines have been prepared and their antifungal, ${ }^{1}$ antiprotozoal, ${ }^{2}$ antibacterial, ${ }^{3}$ anti-HIV, ${ }^{4}$ antithrombotic, ${ }^{5}$ and antidegenerative ${ }^{6}$ potentials have been evaluated. Pentamidine is clinically used for treatment of pneumonia and first stage human African tripanosomiasis. ${ }^{7}$ That drug is on the WHO's List of Essential Medicines for the basic health system. ${ }^{8}$ Moreover, amidine containing molecules have been pointed as proeminent prototypes in the search for new anticancer agents. ${ }^{9}$ In addition,

*e-mail: lucas.pizzuti@gmail.com thiophene-2-carboximidamides have shown potent and selective inhibitory activities of nitric oxide synthases for the treatment of human melanoma. ${ }^{10}$

In the same context, pyrazoles are recognized as a fundamental class of heterocyclic compounds because of their well-established applicability in several areas as agrochemicals, functional materials and medicines. ${ }^{11}$ Dihydro- $1 H$-pyrazole derivatives have been reported as antiproliferative agents ${ }^{12}$ and inhibitors of vascular endothelium growth factors, ${ }^{13}$ mitotic kinesin spindle protein (KSP), ${ }^{14}$ and multidrug resistance protein 1 (MDR1). ${ }^{15}$ Pyrazoline derivatives have shown high selectivity against leukemia cell lines (the concentration for $50 \%$ of maximal inhibition of cell proliferation, $\mathrm{GI}_{50}=0.69-3.35 \mu \mathrm{mol} \mathrm{L}^{-1}$ ) in comparison with eight other tumor cell lines. ${ }^{16}$ 
Taking into account the valuable pharmacological properties of pyrazoline and amidine scaffolds, we envisioned that hybrid molecules could be active against leukemia cells. Analogous 4,5-dihydro- $1 \mathrm{H}$-pyrazole1-carboximidamides have been already prepared by the cyclocondensation of $\alpha, \beta$-unsaturated ketones with aminoguanidine free base under conventional ${ }^{17}$ and sonochemical ${ }^{18}$ conditions. However, to the best of our knowledge there are not reported studies about the antileukemic potency of these hybrid molecules.

Thus, in our continuous efforts to develop sonochemically promoted reactions in environmentally benign solvents ${ }^{19}$ and synthetic methodologies for preparation of heterocyclic compounds ${ }^{20}$ selected on the basis of their biological activity, ${ }^{21}$ we describe herein a rapid and efficient synthetic method for the preparation of 3-(thiophen-2-yl)-4,5dihydro- $1 H$-pyrazole-1-carboximidamide hydrochlorides under ultrasonic conditions. In addition, all compounds synthetized were evaluated against the human Jurkat and RS4;11 acute lymphoblastic leukemia cell lines of T- and B-cell origin, respectively, and the K562 myelogenous leukemia cell line.

\section{Experimental}

Chemistry

\section{General}

3-Aryl-1-(thiophen-2-yl)prop-2-en-1-ones 1a-l were prepared by us following reported procedures. ${ }^{22}$ All the chemicals were used without purification as purchased from commercial suppliers. The sonicated reactions were carried out with a microtip probe connected to a $500 \mathrm{~W}$ Sonics Vibracell ultrasonic processor operating at $20 \mathrm{kHz}$ at $20 \%$ of the maximum power output. Reaction progresses were monitored by gas chromatography (GC). Melting point values were determined in open capillary on an Instrutherm DF-3600 II apparatus and are uncorrected. Infrared spectra (IR) were acquired on a JASCO-4100 spectrophotometer as $\mathrm{KBr}$ pellets. ${ }^{1} \mathrm{H}$ and ${ }^{13} \mathrm{C}$ nuclear magnetic resonance (NMR) spectra were acquired on a Bruker DPX400 instrument (400 MHz for ${ }^{1} \mathrm{H}$ and $101 \mathrm{MHz}$ for ${ }^{13} \mathrm{C}$ ) in $5 \mathrm{~mm}$ sample tubes at $298 \mathrm{~K}$ in dimethyl sulfoxide (DMSO- $d_{6}$ ) using tetramethylsilane (TMS) as internal reference standard. High resolution mass spectra were recorded on a Bruker microTof (Q-TOF) mass spectrometer in electrospray ionization (ESI) mode.

General procedure for the ultrasound-promoted synthesis of 5-aryl-3-(thiophen-2-yl)-4,5-dihydro-1 H-pyrazole-1carboximidamides $(2 \mathbf{a}-\mathbf{I})$
To a $50 \mathrm{~mL}$ vial containing a solution of 3-aryl-1(thiophen-2-yl)prop-2-en-1-ones 1a-l (1 mmol) in ethanol $(15 \mathrm{~mL})$, the aminoguanidine hydrochloride $(0.22 \mathrm{~g}$, $2 \mathrm{mmol})$ and $\mathrm{KOH}(0.11 \mathrm{~g}, 2 \mathrm{mmol})$ were added. The reaction mixture was sonicated for the time indicated in Table 1 and the reaction temperature reached $55-60{ }^{\circ} \mathrm{C}$ after 10 minutes. The resulting solution was cooled to room temperature and acidified using $10 \% \mathrm{HCl}(10-15 \mathrm{~mL})$. The salts were extracted with chloroform $(3 \times 20 \mathrm{~mL})$ and the combined organic layer was dried over anhydrous magnesium sulfate. Removal of solvent under vacuum afforded a crude material. The pure products $\mathbf{2 a}$ - $\mathbf{l}$ were obtained as amorphous solids with yields of $62-95 \%$ after recrystallization from ethyl acetate and drying in a desiccator.

5-Phenyl-3-(thiophen-2-yl)-4,5-dihydro-1 H-pyrazole-1carboximidamide hydrochloride (2a)

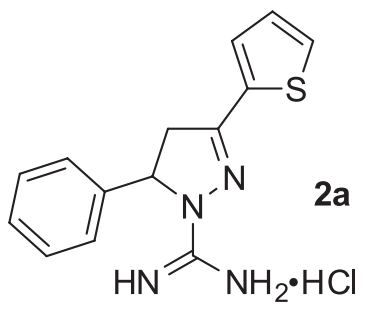

Yellowish solid; $\mathrm{mp}$ 266-268 ${ }^{\circ} \mathrm{C}$; IR ( $\left.\mathrm{KBr}\right)$ $v / \mathrm{cm}^{-1} 3337,3115,1609$, 1430; ${ }^{1} \mathrm{H}$ NMR (400 MHz, DMSO- $\left.d_{6}\right) \delta 7.97$ (bs, 4H, $\left.\mathrm{C}\left(\mathrm{NH}_{2}\right)_{2}\right), 7.86(\mathrm{~d}, 1 \mathrm{H}$, J $\left.5.0 \mathrm{~Hz}, \mathrm{~T}-\mathrm{H}^{*}\right), 7.57$ (d,

$1 \mathrm{H}, J 3.6 \mathrm{~Hz}, \mathrm{~T}-\mathrm{H}), 7.39$ (d, 2H, J 7.6 Hz, Ph-H), 7.35-7.32 (m, 1H, Ph-H), 7.25 (d, 2H, J 7.4 Hz, Ph-H), 7.20-7.17 (m, 1H, T-H), 6.02 (dd, 1H, J 2.4, $11.0 \mathrm{~Hz}, \mathrm{H}_{\mathrm{x}}^{\dagger}$ ) , 4.11 (dd, 1H, $\left.J 11.2,17.8 \mathrm{~Hz}, \mathrm{H}_{\mathrm{m}}^{\dagger}\right), 3.36\left(\mathrm{dd}, 1 \mathrm{H}, J 2.7,17.8 \mathrm{~Hz}, \mathrm{H}_{\mathrm{a}}^{\dagger}\right)$; ${ }^{13} \mathrm{C}$ NMR (101 MHz, DMSO- $d_{6}$ ) $\delta$ 153.2, 153.0, 139.6, 132.7, 132.0, 131.3, 129.0, 128.2, 125.3, 60.4, 44.4; HRMS $\mathrm{m} / \mathrm{z},[\mathrm{M}+\mathrm{H}]^{+}$calcd. for $\mathrm{C}_{14} \mathrm{H}_{15} \mathrm{~N}_{4} \mathrm{~S}$ : 271.1017; found: 271.1015. *T-H: thiophene hydrogens; ${ }^{\dagger} \mathrm{H}_{\mathrm{a}}, \mathrm{H}_{\mathrm{m}}$ and $\mathrm{H}_{\mathrm{x}}$ : pyrazolyl ring hydrogens.

5-(2-Methoxyphenyl)-3-(thiophen-2-yl)-4,5-dihydro-1 Hpyrazole-1-carboximidamide hydrochloride (2b)

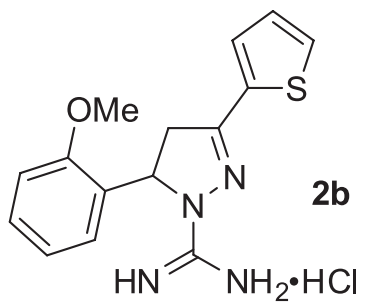

Yellowish solid; mp 287$288{ }^{\circ} \mathrm{C}$; IR $(\mathrm{KBr}) \mathrm{v} / \mathrm{cm}^{-1}$ $3255,3190,3103,1618$, 1423,$1243 ;{ }^{1} \mathrm{H}$ NMR $\left(400 \mathrm{MHz}, \mathrm{DMSO}-d_{6}\right) \delta 7.85$ (bs, 4H, C(NH$\left.)_{2}\right), 7.82$ (dd, $1 \mathrm{H}, J 0.9,5.0 \mathrm{~Hz}, \mathrm{~T}-\mathrm{H}), 7.54$

(dd, 1H, J 0.8, 3.6 Hz, T-H), 7.34 (m, 1H, Ph-H), 7.16 (dd, 1H, J 3.8, 5.0 Hz, T-H), 7.11 (d, 1H, J 8.2 Hz, Ph-H), 6.97-6.89 (m, 2H, Ph-H), 5.87 (dd, 1H, J 3.2, $11.1 \mathrm{~Hz}, \mathrm{H}_{\mathrm{x}}$ ), 4.04 (dd, 1H, J 11.1, 17.7 Hz, H ${ }_{\mathrm{m}}$ ), 3.83 (s, 3H, Ph- $\mathrm{OCH}_{3}$ ), $3.24\left(\mathrm{dd}, 1 \mathrm{H}, J 3.2,17.7 \mathrm{~Hz}, \mathrm{H}_{\mathrm{a}}\right) ;{ }^{13} \mathrm{C} \mathrm{NMR}(101 \mathrm{MHz}$, DMSO- $\left.d_{6}\right) \delta 156.2,153.8,152.9,132.8,131.8,131.1$, 129.6, 128.2, 126.3, 124.9, 120.3, 111.8, 57.2, 55.7, 43.3; 
HRMS $m / z,[\mathrm{M}+\mathrm{H}]^{+}$calcd. for $\mathrm{C}_{15} \mathrm{H}_{17} \mathrm{~N}_{4} \mathrm{OS}$ : 301.1123; found: 301.1148 .

5-(2-Bromophenyl)-3-(thiophen-2-yl)-4,5-dihydro-1Hpyrazole-1-carboximidamide hydrochloride (2c)

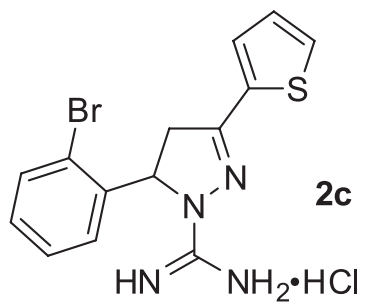

Yellowish solid; $\mathrm{mp}$ 285-288 ${ }^{\circ} \mathrm{C}$; IR (KBr) $\mathrm{V} / \mathrm{cm}^{-1}$ 3290, 3117, 1603, 1423; ${ }^{1} \mathrm{H}$ NMR $(400 \mathrm{MHz}$,

2c DMSO- $\left.d_{6}\right) \delta 8.03(\mathrm{bs}, 4 \mathrm{H}$, $\left.\mathrm{C}\left(\mathrm{NH}_{2}\right)_{2}\right), 7.86(\mathrm{~d}, 1 \mathrm{H}$, $J 4.9 \mathrm{~Hz}, \mathrm{~T}-\mathrm{H}), 7.57(\mathrm{~d}$, $1 \mathrm{H}, J 3.4 \mathrm{~Hz}, \mathrm{~T}-\mathrm{H}), 7.55$ (d, 1H, J $8.1 \mathrm{~Hz}, \mathrm{Ph}-\mathrm{H}), 7.48(\mathrm{~m}, 1 \mathrm{H}, \mathrm{Ph}-\mathrm{H}), 7.38$ (t, $1 \mathrm{H}, J 7.9 \mathrm{~Hz}, \mathrm{Ph}-\mathrm{H}), 7.22-7.18$ (m, 2H, T-H and $\mathrm{Ph}-\mathrm{H})$, $6.04\left(\mathrm{dd}, 1 \mathrm{H}, J 2.8,11.2 \mathrm{~Hz}, \mathrm{H}_{\mathrm{x}}\right), 4.11(\mathrm{dd}, 1 \mathrm{H}, J 11.2$, $\left.17.9 \mathrm{~Hz}, \mathrm{H}_{\mathrm{m}}\right), 3.42\left(\mathrm{dd}, 1 \mathrm{H}, J 2.9,17.9 \mathrm{~Hz}, \mathrm{H}_{\mathrm{a}}\right) ;{ }^{13} \mathrm{C} \mathrm{NMR}$ $\left(101 \mathrm{MHz}\right.$, DMSO- $\left.d_{6}\right) \delta 153.2,153.1,135.2,133.4,132.6$, 132.3, 132.0, 131.3, 129.8, 128.1, 127.6, 126.7, 58.6, 43.2; HRMS $m / z,[\mathrm{M}+\mathrm{H}]^{+}$calcd. for $\mathrm{C}_{14} \mathrm{H}_{14} \mathrm{BrN}_{4} \mathrm{~S}: 349.0122$; found: 349.0129 .

5-(3-Nitrophenyl)-3-(thiophen-2-yl)-4,5-dihydro- $1 \mathrm{H}$ pyrazole-1-carboximidamide hydrochloride (2d)

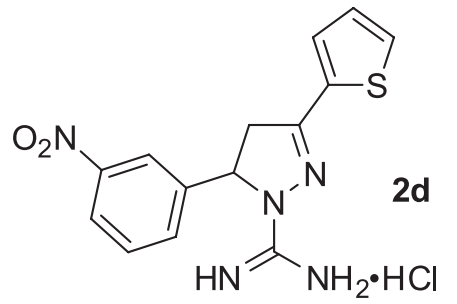

Yellowish solid; mp 288-291 ${ }^{\circ} \mathrm{C}$; IR (KBr) $\mathrm{v} / \mathrm{cm}^{-1} 3363,3284$, 3204, 3103, 1611, 1524, 1423, 1344, 847; ${ }^{1} \mathrm{H}$ NMR $(400 \mathrm{MHz}$, DMSO- $\left.d_{6}\right) \delta 8.22(\mathrm{ddd}$, $1 \mathrm{H}, J$ 0.9, 2.2, $8.2 \mathrm{~Hz}, \mathrm{Ph}-\mathrm{H}), 8.10(\mathrm{t}, 1 \mathrm{H}, J 1.9 \mathrm{~Hz}$, $\mathrm{Ph}-\mathrm{H}), 7.97\left(\mathrm{~s}, 4 \mathrm{H}, \mathrm{C}\left(\mathrm{NH}_{2}\right)_{2}\right), 7.87$ (dd, $1 \mathrm{H}, J 1.1,5.0 \mathrm{~Hz}$, $\mathrm{T}-\mathrm{H}), 7.74$ (t, 1H, J 8.0 Hz, Ph-H), $7.65(\mathrm{~d}, 1 \mathrm{H}, J 7.9 \mathrm{~Hz}$, $\mathrm{Ph}-\mathrm{H}), 7.55$ (dd, $1 \mathrm{H}, J 1.1,3.7 \mathrm{~Hz}, \mathrm{~T}-\mathrm{H}), 7.20(\mathrm{dd}, 1 \mathrm{H}$, $J$ 3.7, $5.0 \mathrm{~Hz}, \mathrm{~T}-\mathrm{H}), 6.07\left(\mathrm{dd}, 1 \mathrm{H}, J 3.2,11.3 \mathrm{~Hz}, \mathrm{H}_{\mathrm{x}}\right.$ ), $4.16\left(\mathrm{dd}, 1 \mathrm{H}, J 11.4,18.0 \mathrm{~Hz}, \mathrm{H}_{\mathrm{m}}\right), 3.48(\mathrm{dd}, 1 \mathrm{H}, J 3.2$, 18.0, $\left.\mathrm{H}_{\mathrm{a}}\right) ;{ }^{13} \mathrm{C}$ NMR $\left(101 \mathrm{MHz}, \mathrm{DMSO}-d_{6}\right) \delta 153.2,152.8$, $148.0,141.5,132.5,132.2,132.0,131.5,130.9,128.2$, 123.2, 120.7, 59.6, 44.3; HRMS $m / z,[\mathrm{M}+\mathrm{H}]^{+}$calcd. for $\mathrm{C}_{14} \mathrm{H}_{14} \mathrm{~N}_{5} \mathrm{O}_{2} \mathrm{~S}$ : 316.0868 ; found: 316.0878 .

5-(4-Methylphenyl)-3-(thiophen-2-yl)-4,5-dihydro- $1 \mathrm{H}$ pyrazole-1-carboximidamide hydrochloride (2e)

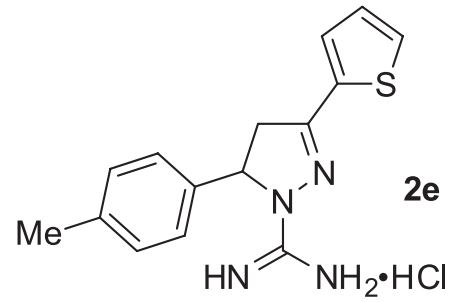

Yellowish solid; mp 278-280 ${ }^{\circ} \mathrm{C}$; IR $(\mathrm{KBr}) \vee / \mathrm{cm}^{-1} 3255$, 3096, 1611, 1430; ${ }^{1} \mathrm{H}$ NMR (400 MHz, DMSO- $\left.d_{6}\right) \delta 7.91$ (bs, $\left.4 \mathrm{H}, \mathrm{C}\left(\mathrm{NH}_{2}\right)_{2}\right), 7.84$ (d,
$1 \mathrm{H}, J 5.0 \mathrm{~Hz}, \mathrm{~T}-\mathrm{H}), 7.56$ (d, 1H, J3.6 Hz, T-H), 7.21-7.17 $(\mathrm{m}, 3 \mathrm{H}, \mathrm{T}-\mathrm{H}$ and $\mathrm{Ph}-\mathrm{H}), 7.13(\mathrm{~d}, 2 \mathrm{H}, J 8.1 \mathrm{~Hz}, \mathrm{Ph}-\mathrm{H})$, $5.92\left(\mathrm{dd}, 1 \mathrm{H}, J 3.0,11.1 \mathrm{~Hz}, \mathrm{H}_{\mathrm{x}}\right.$ ), 4.07 (dd, 1H, $J 11.2$, $\left.17.9 \mathrm{~Hz}, \mathrm{H}_{\mathrm{m}}\right), 3.33$ (dd, 1H, J 3.0, $\left.17.7 \mathrm{~Hz}, \mathrm{H}_{\mathrm{a}}\right), 2.28$ (s, $\left.3 \mathrm{H}, \mathrm{Ph}-\mathrm{CH}_{3}\right) ;{ }^{13} \mathrm{C}$ NMR (101 MHz, DMSO- $\left.d_{6}\right) \delta 153.1$, 153.0, 137.6, 136.6, 132.8, 131.9, 131.2, 129.5, 128.2, 125.3, 60.2, 44.3, 20.6; HRMS $\mathrm{m} / \mathrm{z}$, [M + H] $]^{+}$calcd. for $\mathrm{C}_{15} \mathrm{H}_{17} \mathrm{~N}_{4} \mathrm{~S}$ : 285.1174; found: 285.1179 .

5-(4-Trifluoromethylphenyl)-3-(thiophen-2-yl)-4,5-dihydro$1 \mathrm{H}$-pyrazole-1-carboximidamide hydrochloride (2f)

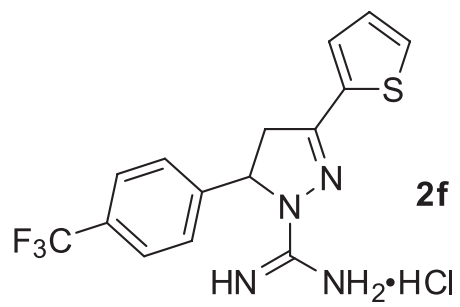

Yellowish solid; mp 295-298 ${ }^{\circ} \mathrm{C}$; IR (KBr) $v / \mathrm{cm}^{-1} 3370$, 3290, 3068, 1603, 1423, 1315-1113, 839; ${ }^{1} \mathrm{H}$ NMR (400 MHz, DMSO- $\left.d_{6}\right) \delta 8.14$ (bs, $\left.4 \mathrm{H}, \mathrm{C}\left(\mathrm{NH}_{2}\right)_{2}\right), 7.88(\mathrm{~d}, 1 \mathrm{H}, J 4.7 \mathrm{~Hz}, \mathrm{~T}-\mathrm{H}), 7.79(\mathrm{~d}, 2 \mathrm{H}$, $J 8.2 \mathrm{~Hz}, \mathrm{Ph}-\mathrm{H}), 7.60(\mathrm{~d}, 1 \mathrm{H}, J 3.1 \mathrm{~Hz}, \mathrm{~T}-\mathrm{H}), 7.52(\mathrm{~d}, 2 \mathrm{H}$, $J 8.1 \mathrm{~Hz}, \mathrm{Ph}-\mathrm{H}), 7.21-7.19(\mathrm{~m}, 1 \mathrm{H}, \mathrm{T}-\mathrm{H}), 6.27$ (dd, $1 \mathrm{H}, J 2.4$, $11.0 \mathrm{~Hz}, \mathrm{H}_{\mathrm{x}}$ ), 4.19 (dd, $1 \mathrm{H}, J 11.3,17.8 \mathrm{~Hz}, \mathrm{H}_{\mathrm{m}}$ ), 3.45 (dd, $\left.1 \mathrm{H}, J 2.8,17.9 \mathrm{~Hz}, \mathrm{H}_{\mathrm{a}}\right) ;{ }^{13} \mathrm{C}$ NMR (101 MHz, DMSO- $\left.d_{6}\right)$ $\delta$ 153.1, 153.0, 143.9 (q, $J 1.3 \mathrm{~Hz}), 132.5,132.0,131.3$, 128.7 (q, J32.0 Hz), 128.1, 126.3, 125.8 (q, J3.8 Hz), 123.9 (q, $J 272.1 \mathrm{~Hz}$ ), 60.0, 44.2; HRMS m/z, [M + H] ${ }^{+}$calcd. for $\mathrm{C}_{15} \mathrm{H}_{14} \mathrm{~F}_{3} \mathrm{~N}_{4} \mathrm{~S}$ : 339.0891 ; found: 339.0881 .

5-(4-Methoxyphenyl)-3-(thiophen-2-yl)-4,5-dihydro-1 Hpyrazole-1-carboximidamide hydrochloride $(2 \mathrm{~g})$

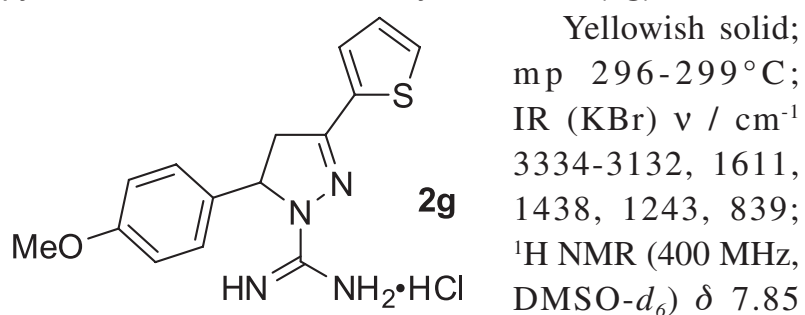

(dd, $1 \mathrm{H}, J 1.0,5.0 \mathrm{~Hz}, \mathrm{~T}-\mathrm{H}$ ), 7.76 (bs, 4H, C( $\left.\left(\mathrm{NH}_{2}\right)_{2}\right), 7.56$ (dd, $1 \mathrm{H}, J 1.1,3.7 \mathrm{~Hz}, \mathrm{~T}-\mathrm{H}), 7.19$ (dd, 1H, J 3.7, $5.0 \mathrm{~Hz}$, $\mathrm{T}-\mathrm{H})$, 7.16-7.09 (m, 2H, Ph-H), 7.01-6.89 (m, 2H, Ph-H), $5.79\left(\mathrm{dd}, 1 \mathrm{H}, J 2.4,11.0 \mathrm{~Hz}, \mathrm{H}_{\mathrm{x}}\right), 4.05(\mathrm{dd}, 1 \mathrm{H}, J 11.1$, $\left.17.8 \mathrm{~Hz}, \mathrm{H}_{\mathrm{m}}\right), 3.73\left(\mathrm{~s}, 3 \mathrm{H}, \mathrm{Ph}-\mathrm{OCH}_{3}\right), 3.41-3.26(\mathrm{~m}, 1 \mathrm{H}$, $\left.\mathrm{H}_{\mathrm{a}}\right) ;{ }^{13} \mathrm{C}$ NMR $\left(101 \mathrm{MHz}\right.$, DMSO- $\left.d_{6}\right) \delta 159.2,153.3,152.7$, 132.8, 132.0, 131.5, 131.3, 128.2, 126.7, 114.4, 60.0, 55.2, 44.4; HRMS $m / z$, $[\mathrm{M}+\mathrm{H}]^{+}$calcd. for $\mathrm{C}_{15} \mathrm{H}_{17} \mathrm{~N}_{4} \mathrm{OS}$ : 301.1123; found: 301.1132 .

5-(4-Fluorophenyl)-3-(thiophen-2-yl)-4,5-dihydro- $1 \mathrm{H}$ pyrazole-1-carboximidamide hydrochloride $(2 \mathrm{~h})$

Yellowish solid; mp 243-245 ${ }^{\circ} \mathrm{C}$; IR (KBr) v / $\mathrm{cm}^{-1}$ 3348-3103, 1596, 1416, 1229, 832; ' ${ }^{1} \mathrm{H}$ NMR (400 MHz, 
<smiles>N=C(N)N1N=C(c2cccs2)CC1c1ccc(F)cc1</smiles>

$\left.\mathrm{DMSO}-d_{6}\right) \delta 7.93(\mathrm{~s}$, $\left.4 \mathrm{H}, \mathrm{C}\left(\mathrm{NH}_{2}\right)_{2}\right), 7.86(\mathrm{dd}$, $1 \mathrm{H}, J 1.1,5.0 \mathrm{~Hz}, \mathrm{~T}-\mathrm{H})$, $7.57(\mathrm{dd}, 1 \mathrm{H}, J 1.1$, $3.7 \mathrm{~Hz}, \mathrm{~T}-\mathrm{H}), 7.33-$ $7.21(\mathrm{~m}, 4 \mathrm{H}, \mathrm{Ph}-\mathrm{H})$, $7.19(\mathrm{dd}, 1 \mathrm{H}, J 3.7$, $5.0 \mathrm{~Hz}, \mathrm{~T}-\mathrm{H}), 5.94\left(\mathrm{dd}, 1 \mathrm{H}, J\right.$ 2.9, $\left.11.2 \mathrm{~Hz}, \mathrm{H}_{\mathrm{x}}\right), 4.09$ (dd, 1H, J 11.2, 17.9 Hz, $\mathrm{H}_{\mathrm{m}}$ ), 3.53-3.27 (m, 1H, $\mathrm{H}_{\mathrm{a}}$ ); ${ }^{13} \mathrm{C}$ NMR (101 MHz, DMSO- $d_{6}$ ) $\delta 163.1,160.6,153.0$ (d, J 34.2 Hz), 135.8 (d, J 2.7 Hz), 132.7, 132.1, 131.4, 128.2, 127.6 (d, J 8.3 Hz), 115.9 (d, J 21.7 Hz), 59.7, 44.4; HRMS $m / z,[\mathrm{M}+\mathrm{H}]^{+}$calcd. for $\mathrm{C}_{14} \mathrm{H}_{14} \mathrm{FN}_{4} \mathrm{~S}$ : 289.0923; found: 289.0936.

5-(4-Chlorophenyl)-3-(thiophen-2-yl)-4,5-dihydro- $1 \mathrm{H}$ pyrazole-1-carboximidamide hydrochloride (2i)

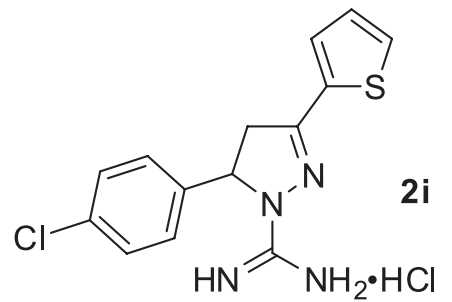

Yellowish solid; mp 257-260 ${ }^{\circ} \mathrm{C}$; IR (KBr) $v / \mathrm{cm}^{-1} 3070$, $1606,1425,825$; 2i ${ }^{1} \mathrm{H}$ NMR $(400 \mathrm{MHz}$, DMSO- $\left.d_{6}\right) \delta 7.98(\mathrm{bs}$, $\left.4 \mathrm{H}, \mathrm{C}\left(\mathrm{NH}_{2}\right)_{2}\right), 7.85$ (d,

1H, J $4.8 \mathrm{~Hz}, \mathrm{~T}-\mathrm{H}), 7.56$ (d, 1H, J $3.1 \mathrm{~Hz}, \mathrm{~T}-\mathrm{H}), 7.47$ (d, 2H, J $8.2 \mathrm{~Hz}, \mathrm{Ph}-\mathrm{H}), 7.27$ (d, 2H, J $8.3 \mathrm{~Hz}, \mathrm{Ph}-\mathrm{H})$, 7.20-7.18 (m, 1H, T-H), 6.01-5.99 (m, 1H, $\left.\mathrm{H}_{\mathrm{x}}\right), 4.10$ (dd, 1H, J 11.2, $\left.17.8 \mathrm{~Hz}, \mathrm{H}_{\mathrm{m}}\right), 3.40-3.35\left(\mathrm{~m}, 1 \mathrm{H}, \mathrm{H}_{\mathrm{a}}\right)$; ${ }^{13} \mathrm{C}$ NMR (101 MHz, DMSO- $\left.d_{6}\right) \delta 153.2,152.9,138.5$, 132.9, 132.6, 132.1, 131.4, 129.0, 128.2, 127.4, 59.8, 44.3; HRMS $m / z,[\mathrm{M}+\mathrm{H}]^{+}$calcd. for $\mathrm{C}_{14} \mathrm{H}_{14} \mathrm{ClN}_{4} \mathrm{~S}$ : 305.0628; found: 305.0629 .

5-(4-Bromophenyl)-3-(thiophen-2-yl)-4,5-dihydro- $1 \mathrm{H}$ pyrazole-1-carboximidamide hydrochloride (2j)

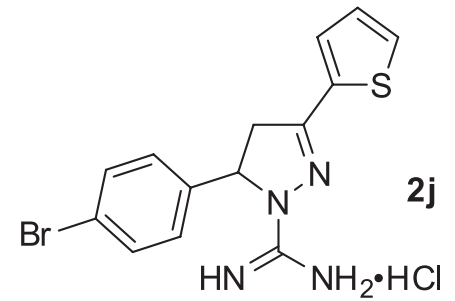

Yellowish solid; $\mathrm{mp}$ 265-266 ${ }^{\circ} \mathrm{C}$; IR (KBr) $\mathrm{v} / \mathrm{cm}^{-1} 3269,3190$, $3103,1611,1430$;

j ${ }^{1} \mathrm{H}$ NMR $(400 \mathrm{MHz}$ DMSO- $\left.d_{6}\right) \delta 8.01(\mathrm{bs}$ $\left.4 \mathrm{H}, \mathrm{C}\left(\mathrm{NH}_{2}\right)_{2}\right), 7.85(\mathrm{~d}$, 1H, J 5.0 Hz, T-H), 7.60 (d, 2H, J $8.3 \mathrm{~Hz}, \mathrm{Ph}-\mathrm{H}), 7.56$ (d, $1 \mathrm{H}, J 3.6 \mathrm{~Hz}, \mathrm{~T}-\mathrm{H}), 7.22$ (d, 2H, J 8.4 Hz, Ph-H), 7.20-7.18 (m, 1H, T-H), 6.03 (dd, 1H, J 2.6, $11.1 \mathrm{~Hz}, \mathrm{H}_{\mathrm{x}}$ ), 4.11 (dd, $\left.1 \mathrm{H}, J 11.3,17.8 \mathrm{~Hz}, \mathrm{H}_{\mathrm{m}}\right), 3.38$ (dd, $1 \mathrm{H}, J 2.7,17.8 \mathrm{~Hz}, \mathrm{H}_{\mathrm{a}}$ ); ${ }^{13} \mathrm{C}$ NMR (101 MHz, DMSO- $\left.d_{6}\right) \delta$ 153.0, 152.9, 138.8, 132.5, 131.9, 131.8, 131.2, 128.1, 127.6, 121.3, 59.7, 44.1; HRMS $m / z,[\mathrm{M}+\mathrm{H}]^{+}$calcd. for $\mathrm{C}_{14} \mathrm{H}_{15} \mathrm{~N}_{4} \mathrm{SBr}$ : 349.0122; found: 349.0141 .
5-(2,4-Dichlorophenyl)-3-(thiophen-2-yl)-4,5-dihydro- $1 \mathrm{H}$ pyrazole-1-carboximidamide hydrochloride (2k)

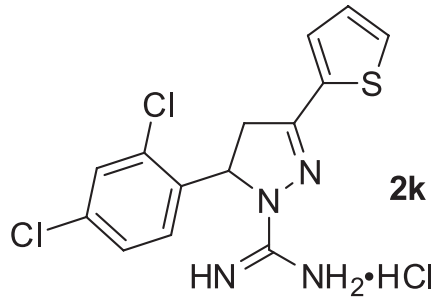

Yellowish solid; $\mathrm{mp}$ 290-293 ${ }^{\circ} \mathrm{C}$; IR (KBr) $\mathrm{V} / \mathrm{cm}^{-1} 3334,3175$, 3103, 1611, 1423, 825; ${ }^{1} \mathrm{H}$ NMR $(400 \mathrm{MHz}$, DMSO- $\left.d_{6}\right) \delta 8.00(\mathrm{bs}$, $\left.4 \mathrm{H}, \mathrm{C}\left(\mathrm{NH}_{2}\right)_{2}\right), 7.85(\mathrm{dd}$,

$1 \mathrm{H}, J 1.1,5.0 \mathrm{~Hz}, \mathrm{~T}-\mathrm{H}), 7.56(\mathrm{dd}, 1 \mathrm{H}, J 1.1,3.7 \mathrm{~Hz}, \mathrm{~T}-\mathrm{H})$, 7.55-7.53 (m, 1H, Ph-H), 7.48-7.47 (m, 1H, Ph-H), 7.38 (t, $1 \mathrm{H}, J 7.9 \mathrm{~Hz}, \mathrm{Ph}-\mathrm{H}), 7.21-7.18$ (m, 2H, T-H and $\mathrm{Ph}-\mathrm{H})$, 6.02 (dd, 1H, J 3.1, $11.2 \mathrm{~Hz}, \mathrm{H}_{\mathrm{x}}$ ), 4.10 (dd, 1H, J 11.3, $\left.17.9 \mathrm{~Hz}, \mathrm{H}_{\mathrm{m}}\right), 3.42$ (dd, 1H, J 3.1, $17.9 \mathrm{~Hz}, \mathrm{H}_{\mathrm{a}}$ ); ${ }^{13} \mathrm{C} \mathrm{NMR}$ $\left(101 \mathrm{MHz}, \mathrm{DMSO}-d_{6}\right) \delta 153.1,152.9,142.0,132.5,132.0$, 131.2, 131.2, 131.0, 128.4, 128.1, 124.1, 121.9, 59.6, 44.2; HRMS $m / z,[\mathrm{M}+\mathrm{H}]^{+}$calcd. for $\mathrm{C}_{14} \mathrm{H}_{13} \mathrm{Cl}_{2} \mathrm{~N}_{4} \mathrm{~S}$ : 339.0238; found: 339.0237 .

5-(3,4-Dimethoxyphenyl)-3-(thiophen-2-yl)-4,5-dihydro- $1 \mathrm{H}$ pyrazole-1-carboximidamide hydrochloride (2I)

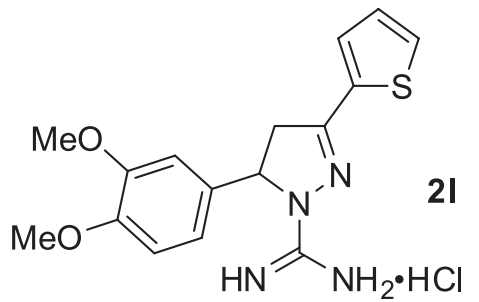

Yellowish solid; $\operatorname{mp~287-291~}{ }^{\circ} \mathrm{C}$; IR $(\mathrm{KBr}) \vee / \mathrm{cm}^{-1}$ 3298, 3139, 2830, $1618, \quad 1430$; ${ }^{1} \mathrm{H}$ NMR $(400 \mathrm{MHz}$, $\left.\mathrm{DMSO}-d_{6}\right) \delta 7.84$

(dd, 1H, J 1.0, $5.0 \mathrm{~Hz}, \mathrm{~T}-\mathrm{H}), 7.80$ (bs, 4H, C( $\left.\left.\mathrm{NH}_{2}\right)_{2}\right), 7.56$ (dd, 1H, J 1.0, $3.6 \mathrm{~Hz}, \mathrm{~T}-\mathrm{H}), 7.19$ (dd, 1H, J 3.7, $5.0 \mathrm{~Hz}$, T-H), 6.96 (d, 1H, J 8.3 Hz, Ph-H), 6.92 (d, 1H, J 1.9 Hz, $\mathrm{Ph}-\mathrm{H}), 6.67$ (dd, 1H, J 1.9, $8.3 \mathrm{~Hz}, \mathrm{Ph}-\mathrm{H}), 5.78$ (dd, 1H, $\left.J 2.9,11.1 \mathrm{~Hz}, \mathrm{H}_{\mathrm{x}}\right), 4.05$ (dd, $\left.1 \mathrm{H}, J 11.1,17.8 \mathrm{~Hz}, \mathrm{H}_{\mathrm{m}}\right), 3.75$ (s, 3H, Ph- $\mathrm{OCH}_{3}$ ), 3.73 (s, 3H, Ph- $\mathrm{OCH}_{3}$ ), 3.40-3.36 (m, $\left.1 \mathrm{H}, \mathrm{H}_{\mathrm{a}}\right) ;{ }^{13} \mathrm{C}$ NMR $\left(101 \mathrm{MHz}, \mathrm{DMSO}-d_{6}\right) \delta 153.2,152.8$, $148.9,148.7,132.8,131.8,131.8,131.8,131.1,128.2$, $116.9,112.3,110.1,60.3,55.6,44.4 ; \mathrm{HRMS} m / z,[\mathrm{M}+\mathrm{H}]^{+}$ calcd. for $\mathrm{C}_{16} \mathrm{H}_{19} \mathrm{~N}_{4} \mathrm{O}_{2} \mathrm{~S}$ : 331.1229; found: 331.1252 .

In vitro citotoxicity assay (MTT assay)

In vitro cytotoxicity assay was carried out on three different human leukemia cell lines: K562, erythroleukemia cells bearing the $\mathrm{t}(9 ; 22)(\mathrm{q} 34 ; \mathrm{q} 11)$-derived BCR/ABL1 fusion gene; Jurkat, T-cells acute lymphoblastic leukemia (ALL) cells; and RS4;11, B-cell precursor ALL cells bearing the $\mathrm{t}(4 ; 11)(\mathrm{q} 21 ; \mathrm{q} 23)$-derived KMT2A/AFF1 fusion gene. The cells were maintained in RPMI-1640 (Cultilab) supplemented with $10 \%$ fetal bovine serum (FBS; Cultilab) and penicillin/streptomycin, at $37{ }^{\circ} \mathrm{C}$ and $5 \% \mathrm{CO}_{2}$. For the cytotoxicity assay, compounds $\mathbf{2 a - 1}$ were dissolved in 
DMSO in order to obtain a stock solution of $20 \mathrm{mmol} \mathrm{L}^{-1}$. Further dilutions were made in complete culture medium immediately before use. K562 and Jurkat cells were seeded at $3 \times 10^{4}$ cells per well while RS4;11 cells were seeded at $4 \times 10^{4}$ cells per well in a 96-well plate. After that, $20 \mu \mathrm{L}$ of 10 -fold serial dilutions of compounds $\mathbf{2 a - 1}$ or vehicle (DMSO at a final concentration of $0.5 \%$ ) were added to each well, in triplicate, at final concentrations of $0.1,1.0$, 10 and $100 \mu \mathrm{mol} \mathrm{L} \mathrm{L}^{-1}$. Culture plates were kept at $37^{\circ} \mathrm{C}$ and $5 \% \mathrm{CO}_{2}$ for $48 \mathrm{~h}$, then, cell viability was measured by adding $20 \mu \mathrm{L}$ of the MTT (3-(4,5-dimethylthiazol-2-yl)2,5-diphenyltetrazolium bromide) reagent (Sigma-Aldrich) at $5 \mathrm{mg} \mathrm{mL}^{-1}$. After $4 \mathrm{~h}$, the precipitated formazan crystals were dissolved by the addition of $100 \mu \mathrm{L}$ of an acid sodium dodecyl sulfate solution (10\% SDS, $0.01 \mathrm{~mol} \mathrm{~L}^{-1} \mathrm{HCl}$ ). Following overnight incubation, absorbance was measured at $570 \mathrm{~nm}$ for the MTT reaction and $620 \mathrm{~nm}$ as reference on scanning. Viability was calculated as a percentage of viable cells at different test concentrations relative to the control (vehicle-treated) cells. The concentration of compounds 2a-1 that resulted in $50 \%$ inhibition of cell growth was calculated as the half maximal inhibitory concentration $\left(\mathrm{IC}_{50}\right)$ by constructing a dose-response curve using GraphPad Prism 5 software (GraphPad Software, Inc., La Jolla, CA).

\section{Results and Discussion}

\section{Chemistry}

The 4,5-dihydro- $1 H$-pyrazole-1-carboximidamides (2a-1) were synthesized in good yields by the ultrasoundassisted cyclocondensation reaction between compounds 1a-l, prepared as described in literature, ${ }^{22}$ and aminoguanidine hydrochloride in the presence of $\mathrm{KOH}$ using ethanol as a green solvent (Table 1). The time required for the completion of the reaction depends on the nature of the groups attached to the benzene ring and it was determined by monitoring de consumption of the carbonyl compound by gas chromatography (GC) in intervals of 5 minutes. After the total consumption of the starting materials 1a-l the reaction solution was acidified with $\mathrm{HCl}$ in order to obtain the salts of the products. As showed in Table 1, the reaction tolerate electron-withdrawing and electron-donating groups. In general, substrates that contain electron-withdrawing substituents such as $3-\mathrm{NO}_{2}$ (Table 1 , entry 4) and $4-\mathrm{CF}_{3}$ (Table 1 , entry 6 ) gave products in shorter reaction times but in lower yields.

The structure of the carboximidamides were confirmed by IR, ${ }^{1} \mathrm{H}$ and ${ }^{13} \mathrm{C}$ NMR and HRMS. The IR spectra showed

Table 1. Cyclocondensation reactions between 1a-l and aminoguanidine hydrochloride in the presence of KOH for the preparation of carboximidamides $2 \mathrm{a}-\mathrm{I}$<smiles></smiles>

\begin{tabular}{lccccccc}
\hline entry & Product & $\mathrm{R}^{1}$ & $\mathrm{R}^{2}$ & $\mathrm{R}^{3}$ & $\mathrm{R}^{4}$ & time $^{\text {a }}$ min & Yield $^{\text {b } \%}$ \\
\hline 1 & $\mathbf{2 a}$ & $\mathrm{H}$ & $\mathrm{H}$ & $\mathrm{H}$ & $\mathrm{H}$ & 20 & 88 \\
2 & $\mathbf{2 b}$ & $\mathrm{OMe}$ & $\mathrm{H}$ & $\mathrm{H}$ & $\mathrm{H}$ & 25 & 80 \\
3 & $\mathbf{2 c}$ & $\mathrm{Br}$ & $\mathrm{H}$ & $\mathrm{H}$ & $\mathrm{H}$ & 30 & 70 \\
4 & $\mathbf{2 d}$ & $\mathrm{H}$ & $\mathrm{NO}_{2}$ & $\mathrm{H}$ & $\mathrm{H}$ & 15 & 65 \\
5 & $\mathbf{2 e}$ & $\mathrm{H}$ & $\mathrm{H}$ & $\mathrm{Me}$ & $\mathrm{H}$ & 35 & 80 \\
6 & $\mathbf{2}$ & $\mathrm{H}$ & $\mathrm{H}$ & $\mathrm{CF}_{3}$ & $\mathrm{H}$ & 15 & 62 \\
7 & $\mathbf{2 g}$ & $\mathrm{H}$ & $\mathrm{H}$ & $\mathrm{OMe}$ & $\mathrm{H}$ & 25 & 86 \\
8 & $\mathbf{2 h}$ & $\mathrm{H}$ & $\mathrm{H}$ & $\mathrm{F}$ & $\mathrm{H}$ & 30 & 73 \\
9 & $\mathbf{2}$ & $\mathrm{H}$ & $\mathrm{H}$ & $\mathrm{Cl}$ & $\mathrm{H}$ & 30 & 71 \\
10 & $\mathbf{2}$ & $\mathrm{H}$ & $\mathrm{H}$ & $\mathrm{Br}$ & $\mathrm{H}$ & 30 & 75 \\
11 & $\mathbf{2}$ & $\mathrm{Cl}$ & $\mathrm{H}$ & $\mathrm{Cl}$ & $\mathrm{H}$ & 30 & 66 \\
12 & $\mathbf{2}$ & $\mathrm{H}$ & $\mathrm{OMe}$ & $\mathrm{OMe}$ & $\mathrm{H}$ & 25 & 83 \\
\hline
\end{tabular}

a Sonication time; byields of the isolated compounds. 
sets of absorption bands in accordance with the proposed structures. In the ${ }^{1} \mathrm{H}$ NMR spectra of the compounds, the characteristic signals of the AMX coupling system between pyrazolyl ring hydrogens, $\mathrm{H}_{\mathrm{a}}, \mathrm{H}_{\mathrm{m}}$ and $\mathrm{H}_{\mathrm{x}}$, were observed as sets of three doublets of doublets in the $\delta 3.48$ 3.24 , 4.19-4.04, and 6.27-5.78 ppm regions, respectively. Besides, the formation of the salts was confirmed by the presence of broad singlets in the range of $\delta 8.14-7.76 \mathrm{ppm}$ due to the presence of four equivalent hydrogens attached to nitrogens atoms in the carboximidamidyl moieties. The analysis of the ${ }^{13} \mathrm{C}$ NMR and HRMS spectra also confirmed the structure of the products.

Although the mechanism of the cyclocondensation reaction between aminoguanidine and $\alpha, \beta$-unsaturated ketones was not yet experimentally established, the literature shows a possible explanation based on the hard and soft acids and bases (HSAB) concept and quantum chemical studies on the aminoguanidine. ${ }^{17,23}$ Accordingly, a proposed mechanism is shown in Figure 1. Firstly, $\mathrm{KOH}$ neutralizes the aminoguanidine salt to give aminoguanidine in its free form, which can exist as two tautomers. Thus Aza-Michael-type addition from the internal imine nitrogen to the $\beta$-position of $\mathbf{1}$ leads to adduct $\mathbf{I}$. In the next step, an intramolecular nucleophilic attack of the primary amino nitrogen to the carbonyl leads to intermediates II, which is dehydrated to give the product III. Finally, the addition of $\mathrm{HCl}$ produces the salt $\mathbf{2}$.

\section{In vitro evaluation of antileukemia activity}

To gain insight on structure-activity relationship, twelve pyrazoline derivatives (2a-1) had their cytotoxic activity evaluated against three different human leukemia cell lines (Table 2). Compounds $\mathbf{2 b}, \mathbf{2 c}, \mathbf{2 f}, \mathbf{2} \mathbf{i}, \mathbf{2} \mathbf{j}$ and $\mathbf{2 k}$ showed the best cytotoxic activity, with $\mathrm{IC}_{50}$ value around $15 \mu \mathrm{mol} \mathrm{L}-1$ for at least one of the three leukemia cell lines analyzed. Compounds $\mathbf{2 a}, \mathbf{2 d}, \mathbf{2 e}, \mathbf{2 g}$ and $\mathbf{2 h}$ showed intermediate cytotoxic activity, with $\mathrm{IC}_{50}$ around $40 \mu \mathrm{mol} \mathrm{L}{ }^{-1}$. Finally, compound $2 \mathbf{l}$ did not present a significant cytotoxic activity, with $\mathrm{IC}_{50}$ value $>100 \mu \mathrm{mol} \mathrm{L}-1$.

The in vitro cytotoxic activity of pyrazolines is in the same range to that of several anti-leukemia drugs used in the clinic: 6-mercaptopurine (20.9 and $233.4 \mu \mathrm{mol} \mathrm{L} \mathrm{L}^{-1}$, median $\mathrm{IC}_{50}$ values for a panel of seven $\mathrm{T}$-cell and fifteen B-cell precursor ALL cell lines, respectively), 6-thioguanine (4 and $11.8 \mu \mathrm{mol} \mathrm{L}^{-1}$ ), dexamethasone (82.7

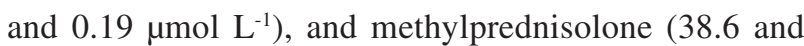
$\left.69.8 \mu \mathrm{mol} \mathrm{L}^{-1}\right) .{ }^{24}$ In conclusion, the cytotoxic activity of most pyrazolines is comparable to that of drugs used in leukemia treatment.

\section{Conclusions}

Ultrasound irradiation was efficient to promote the cyclocondensation reaction between thiophenylchalcones

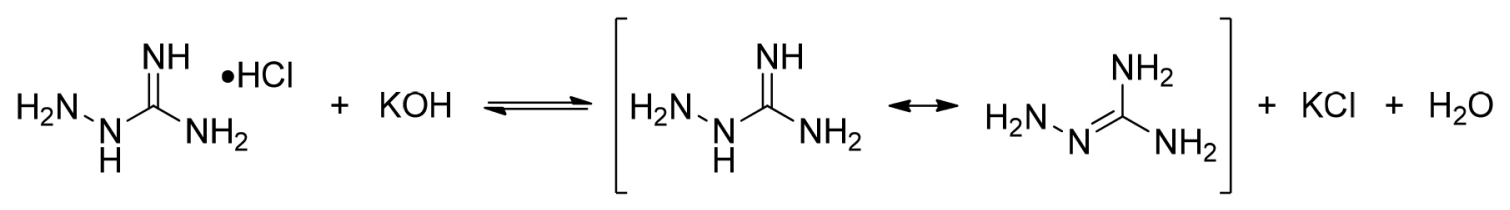<smiles>[R][R]c1ccc(C(CC(=O)c2cccs2)N(N)C(=N)N)cc1</smiles><smiles>[R][R]c1ccc(C2CC(O)(c3cccs3)NN2C(=N)N)cc1</smiles>

Figure 1. Proposed mechanism for the cyclocondensation reaction between 3-aryl-1-(thiophen-2-yl)prop-2-en-1-ones and aminoguanidine hydrochloride in the presence of $\mathrm{KOH}$. 
Table 2. Cytotoxic activity of compounds 2a-l against different leukemia cell lines

\begin{tabular}{lcccc}
\hline \multirow{2}{*}{ entry } & Compound & \multicolumn{3}{c}{$\mathrm{IC}_{50}{ }^{a} /\left(\mu \mathrm{mol} \mathrm{L}{ }^{-1}\right)$} \\
\cline { 3 - 5 } 1 & $\mathbf{2 a}$ & 29.5 & 70.5 & 21.26 \\
\hline 2 & $\mathbf{2 b}$ & 10.2 & 29.4 & 5.9 \\
3 & $\mathbf{2 c}$ & 21.5 & 20.5 & 14.7 \\
4 & $\mathbf{2 d}$ & 29.5 & 46.4 & 33.4 \\
5 & $\mathbf{2 e}$ & 34.2 & 28.7 & 24.8 \\
6 & $\mathbf{2 f}$ & 24.7 & 17.3 & 17.4 \\
7 & $\mathbf{2 g}$ & 28.6 & 47.1 & 40.0 \\
8 & $\mathbf{2 h}$ & 42.4 & 51.5 & 36.2 \\
9 & $\mathbf{2 i}$ & 37.9 & 16.7 & 30.4 \\
10 & $\mathbf{2 j}$ & 22.0 & 14.4 & 23.7 \\
11 & $\mathbf{2 k}$ & 21.3 & 16.2 & 5.7 \\
12 & $\mathbf{2 l}$ & $>100$ & $>100$ & $>100$ \\
\hline
\end{tabular}

aThe drug concentration resulting in a 50\% of maximal inhibition of cell proliferation (as measured by MTT staining).

and aminoguanidine hydrochloride in the presence of $\mathrm{KOH}$, furnishing a series of twelve 3-(thiophen-2-yl)-4,5-dihydro$1 H$-pyrazole-1-carboximidamides in short reaction times (15-35 min) and good yields (62-95\%). All compounds prepared were evaluated against the human Jurkat and RS4;11 acute lymphoblastic leukemia cell lines of T- and B-cell origin, respectively, and the K562 myelogenous leukemia cell line. Six compounds presented $\mathrm{IC}_{50}$ values around $15 \mu \mathrm{mol} \mathrm{L} \mathrm{L}^{-1}$ and five compounds presented $\mathrm{IC}_{50}$ values around $40 \mu \mathrm{mol} \mathrm{L} \mathrm{L}^{-1}$ for at least one of the three cell lines analyzed. One compound was not significantly cytotoxic presenting $\mathrm{IC}_{50}$ value $>100 \mu \mathrm{mol} \mathrm{L}-1$.

\section{Supplementary Information}

Supplementary information $\left({ }^{1} \mathrm{H}\right.$ NMR, ${ }^{13} \mathrm{C}$ NMR and HRMS spectra and dose-response curves) is available free of charge at http://jbcs.sbq.org.br as a PDF file.

\section{Acknowledgments}

We acknowledge Conselho Nacional de Desenvolvimento Científico e Tecnológico (CNPq, grant 483021/2013-0 for L. Pizzuti), Fundação de Apoio ao Desenvolvimento do Ensino, Ciência e Tecnologia do Estado de Mato Grosso do Sul (FUNDECT, grant 0180/12 for L. Pizzuti), and Fundação de Amparo à Pesquisa do Estado de São Paulo (FAPESP, grant 12/12802-1 for J. A. Yunes) for financial support. We also thank Coordenação de Aperfeiçoamento de Pessoal de Nível Superior (CAPES) and FAPESP for scholarships to E. F. S. dos Santos and N. M. Cury, respectively. J. A. Yunes thanks CNPq for the productivity fellowship.

\section{References}

1. Manetti, F.; Castagnolo, D.; Raffi, F.; Zizzari, A. T.; Rajamaki, S.; D’Arezzo, S.; Visca, P.; Cona, A.; Fracasso, M. E.; Doria, D.; Posteraro, B.; Sanguinetti, M.; Fadda, G.; Botta, M.; J. Med. Chem. 2009, 52, 7376.

2. dos Santos, M. S.; Gomes, A. O.; Bernardino, A. M. R.; de Souza, M. C.; Khan, M. A.; de Brito, M. A.; Castro, H. C.; Abreu, P. A.; Rodrigues, C. R.; de Léo, R. M. M.; Leon, L. L.; Canto-Cavalheiro, M. M.; J. Braz. Chem. Soc. 2011, 22, 352; Soeiro, M. N. C.; Werbovetz, K.; Boykin, D. W.; Wilson, W. D.; Wang, M. Z.; Hemphill, A.; Parasitology 2013, 140, 929.

3. Gobis, K.; Foks, H.; Wiśniewska, K.; Dąbrowska-Szponar, M.; Augustynowicz-Kopeć, E.; Napiórkowska, A.; Arch. Pharm. Chem. Life Sci. 2012, 345, 911; Nguyen, S. T.; Williams, J. D.; Butler, M. M.; Ding, X.; Mills, D. M.; Tashjian, T. F.; Panchal, R. G.; Weir, S. K.; Moon, C.; Kim, H.-O.; Marsden, J. A.; Peet, N. P.; Bowlin, T. L.; Bioorg. Med. Chem. Lett. 2014, 24, 3366; Stolić, I.; Paljetak, H. Č.; Perić, M.; Matijašić, M.; Stepanić, V.; Verbanac, D.; Bajić, M.; Eur. J. Med. Chem. 2015, 90, 68.

4. Echevarria, A.; Santos, L. H.; Miller, J.; Mahmood, N.; Bioorg. Med. Chem. Lett. 1996, 6, 1901.

5. Liebeschuetz, J. W.; Jones, S. D.; Morgan, P. J.; Murray, C. W.; Rimmer, A. D.; Roscoe, J. M. E.; Waszkowycz, B.; Welsh, P. M.; Wylie, W. A.; Young, S. C.; Martin, H.; Mahler, J.; Brady, L.; Wilkinson, K.; J. Med. Chem. 2002, 45, 1221.

6. Panico, A.; Vicini, P.; Incert, M.; Cardile, V.; Gentile, B.; Ronsisvalle, G.; Farmaco 2002, 57, 671; Vicini, P.; Incerti, M.; Cardile, V.; Garufi, F.; Ronsisvalle, S.; Panico, A. M.; ChemMedChem 2007, 2, 113; Woltering, T. J.; Wostl, W.; Hilpert, H.; Rogers-Evans, M.; Pinard, E.; Mayweg, A.; Göbel, M.; Banner, D. W.; Benz, J.; Travagli, M.; Pollastrini, M.; Marconi, G.; Gabellieri, E.; Guba, W.; Mauser, H.; Andreini, M.; Jacobsen, H.; Power, E.; Narquizian, R.; Bioorg. Med. Chem. Lett. 2013, 23, 4239;

7. Huang, T. L.; Eynde, J. J. V.; Mayence, A.; Collins, M. S.; Cushion, M. T.; Rattendi, D.; Londono, I.; Mazumder, L.; Bacchi, C. J.; Yarlett, N.; Bioorg. Med. Chem. Lett. 2009, 19, 5884.

8. http://www.who.int/medicines/publications/essentialmedicines/ en/, accessed in May 2016.

9. Bhongade, B. A.; Gadad, A. K.; Bioorg. Med. Chem. 2004, 12, 2797; Özdemir, A.; Altıntop, M. D.; Kaplancıklı, Z. A.; TuranZitouni, G.; Çiftçi, G. A.; Yıldırım, Ş. U.; J. Enzyme Inhib. Med. Chem. 2013, 28, 1221; Houck, J. D.; Dawson, T. K.; Kennedy, A. J.; Kharel, Y.; Naimon, N. D.; Field, S. D.; Lynch, 
K. R.; Macdonald, T. L.; ACS Med. Chem. Lett., in press, DOI: 10.1021/acsmedchemlett.6b00002.

10. Huang, H.; Li, H.; Yang, S.; Chreifi, G.; Martásek, P.; Roman, L. J.; Meyskens, F. L.; Poulos, T. L.; Silverman, R. B.; J. Med. Chem. 2014, 57, 686.

11. Küçükgüzel, S. G.; Şenkardeş, S.; Eur. J. Med. Chem. 2015, 97 , 786; Dias, D.; Pacheco, B. S.; Cunico, W.; Pizzuti, L.; Pereira, C. M. P.; Mini-Rev. Med. Chem. 2014, 14, 1078; Kumar, H.; Saini, D.; Jain, S.; Jain, N.; Eur. J. Med. Chem. 2013, 70, 248; Alex, J. M.; Kumar, R.; J. Enzyme Inhib. Med. Chem. 2014, $29,427$.

12. Johnson, M.; Younglove, B.; Lee, L.; LeBlanc, R.; Holt Jr., H.; Hills, P.; Mackay, H.; Brown, T.; Mooberry, S. L.; Lee, M.; Bioorg. Med. Chem. Lett. 2007, 17, 5897; Havrylyuk, D.; Zimenkovsky, B.; Vasylenko, O.; Zaprutko, L.; Gzella, A.; Lesyk, R.; Eur. J. Med. Chem. 2009, 44, 1396; Shaharyar, M.; Abdullah, M. M.; Bakht, M. A.; Majeed, J.; Eur. J. Med. Chem. 2010, 45, 114; Insuasty, B.; Tigreros, A.; Orozco, F.; Quiroga, J.; Abonıa, R.; Nogueras, M.; Sanchez, A.; Cobo, J.; Bioorg. Med. Chem. 2010, 18, 4965.

13. Kumar, S.; Bawa, S.; Drabu, S.; Kumar, R.; Gupta, H.; Recent Pat. Anti-Infect. Drug Discovery 2009, 4, 154.

14. Roecker, A. J.; Coleman, P. J.; Mercer, S. P.; Schreier, J. D.; Buser, C. A.; Walsh, E. S.; Hamilton, K.; Lobell, R. B.; Tao, W.; Diehl, R. E.; South, V. J.; Davide, J. P.; Kohl, N. E.; Yan, Y.; Kuo, L. C.; Li, C.; Fernandez-Metzler, C.; Mahan, E. A.; Prueksaritanont, T.; Hartman, G. D.; Bioorg. Med. Chem. Lett. 2007, 17, 5677.

15. Kobayashi, H.; Dorai, T.; Holand, J. F.; Ohnuma, T.; Cancer Res. 1994, 54, 1271; Manna, F.; Chimenti, F.; Bolasco, A.; Secci, D.; Bizzarri, B.; Befani, O.; Turini, P.; Mondovi, B.; Alcaro, S.; Tafi, A.; Bioorg. Med. Chem. Lett. 2002, 12, 3629.

16. Havrylyuk, D.; Kovach, N.; Zimenkovsky, B.; Vasylenko, O.; Lesyk, R.; Arch. Pharm. (Weinheim, Ger.) 2011, 344, 514.

17. Světlík, J.; Šallai, L.; J. Heterocycl. Chem. 2002, 39, 363.
18. Pizzuti, L.; Martins, P. L. G.; Ribeiro, B. A.; Quina, F. H.; Pinto, E.; Flores, A. F. C.; Venzke, D.; Pereira, C. M. P.; Ultrason. Sonochem. 2010, 17, 33.

19. Ferreira, I. M.; Casagrande, G. A.; Pizzuti, L.; Raminelli, C.; Synth. Commun. 2014, 44, 2094; Franco, M. S. F.; Casagrande, G. A.; Raminelli, C.; Moura, S.; Rossatto, M.; Quina, F. H.; Pereira, C. M. P.; Flores, A. F. C.; Pizzuti, L.; Synth. Commun. 2015, 45, 692; Kuhn, B. P.; Malavolta, J. L.; Casagrande, G. A.; Raminelli, C.; Quina, F. H.; Pereira, C. M. P.; Flores, A. F. C.; Pizzuti, L.; J. Braz. Chem. Soc. 2015, 26, 1306.

20. Flores, A. F. C.; Pizzuti, L.; Piovesan, L. A.; Flores, D. C.; Malavolta, J. L.; Pereira, C. M. P.; Tetrahedron Lett. 2010, 51, 4908; Flores, A. F. C.; Piovesan, L. A.; Pizzuti, L.; Flores, D. C.; Malavolta, J. L.; Martins, M. A. P.; J. Heterocycl. Chem. 2014, 51, 733 .

21. Silva, F. A. N.; Pizzuti, L.; Quina, F. H.; Souza, S. P.; Rosales, P. F.; Siqueira, G. M.; Pereira, C. P. M.; Barros, S. B. M.; Rivelli, D. P.; Lett. Drug Des. Discovery 2010, 7, 657; Oliveira, S.; Pizzuti, L.; Quina, F.; Flores, A.; Lund, R.; Lencina, C.; Pacheco, B. S.; Pereira, C. M. P.; Piva, E.; Molecules 2014, 19, 5806.

22. Li, R.; Kenyon, G. L.; Cohen, F. E.; Chen, X.; Gong, B.; Dominguez, J. N.; Davidson, E.; Kurzban, G.; Miller, R. E.; Nuzum, E. O.; Rosenthal, P. J.; McKerrow, J. H.; J. Med. Chem. 1995, 38, 5031.

23. Koskinena, J. T.; Koskinen, M.; Mutikainen, I.; Tilus, P.; Mannfors, B.; Elo, H.; Z. Naturforsch. 1997, 52B, 1259.

24. Beesley, A. H.; Palmer, M. L.; Ford, J.; Weller, R. E.; Cummings, A. J.; Freitas, J. R.; Firth, M. J.; Perera, K. U.; de Klerk, N. H.; Kees, U. R.; Br. J. Cancer 2006, 95, 1537.

Submitted: March 3, 2016

Published online: May 31, 2016

FAPESP has sponsored the publication of this article. FAPERGS/CAPES has sponsored the publication of this article. 